\title{
Target Identification Using Harmonic Wavelet Based ISAR Imaging
}

\author{
B. K. Shreyamsha Kumar, B. Prabhakar, K. Suryanarayana, V. Thilagavathi, and R. Rajagopal
}

Central Research Laboratory, Bharat Electronics Limited, Bangalore-560013, India

Received 30 April 2005; Revised 21 November 2005; Accepted 23 November 2005

\begin{abstract}
A new approach has been proposed to reduce the computations involved in the ISAR imaging, which uses harmonic wavelet(HW) based time-frequency representation (TFR). Since the HW-based TFR falls into a category of nonparametric time-frequency (T-F) analysis tool, it is computationally efficient compared to parametric T-F analysis tools such as adaptive joint time-frequency transform (AJTFT), adaptive wavelet transform (AWT), and evolutionary AWT (EAWT). Further, the performance of the proposed method of ISAR imaging is compared with the ISAR imaging by other nonparametric T-F analysis tools such as short-time Fourier transform (STFT) and Choi-Williams distribution (CWD). In the ISAR imaging, the use of HW-based TFR provides similar/better results with significant (92\%) computational advantage compared to that obtained by CWD. The ISAR images thus obtained are identified using a neural network-based classification scheme with feature set invariant to translation, rotation, and scaling.
\end{abstract}

Copyright ( 2006 Hindawi Publishing Corporation. All rights reserved.

\section{INTRODUCTION}

Inverse synthetic aperture radar (ISAR) is an imaging radar that uses the target's pitch, roll, and yaw motions to generate an image in the range-Doppler plane. Primarily, the Fourier transform (FT) was used for the ISAR imaging with the assumption that Doppler frequency is constant over the imaging time duration $[1,2]$. However, the assumption of constant Doppler frequency is not true as the Doppler frequency varies in time because of the nonuniform motion of the target due to maneuvers. Hence the FT-based method suffers from the disadvantage of image blurring in the final output.

In the last decade, many techniques such as transform domain methods, subaperture methods, and superresolution methods have been applied to obtain the time-varying spectrum in the hope of enhancing image resolution. However, none of them completely resolved the blurring problem. With the intention of obtaining focused ISAR image, Chen et al. introduced time-frequency (T-F) transform in the place of FT. Well-known T-F transforms include shorttime Fourier transform (STFT), Wigner-Ville distribution (WVD) [1, 2], continuous wavelet transform (CWT) [3], adaptive joint time-frequency transform (AJTFT) [4], adaptive wavelet transform (AWT) [5], and evolutionary AWT (EAWT) [6]. Among these T-F transforms, STFT, WVD, and CWT fall into a category of nonparametric T-F analysis tools whereas AJTFT, AWT, and EAWT fall into a category of parametric T-F analysis tools. The STFT is the best-known and most basic T-F analysis tool, but it suffers from tradeoff between time resolution and frequency resolution. The WVD $[7,8]$ provides better resolution both in time as well as frequency, but has a cross-term problem. The CWT has multiresolution characteristics and it is free from crossterm problem, but its T-F grid is still rigid $[2,6]$. The AWT provides a more flexible T-F grid than the CWT. Further, it is free from resolution problem and cross-term problem, but its accuracy is limited as it uses a bisection search method and fast FT (FFT) for parameter extraction [5]. The AJTFT uses iterative search method to get the adaptive spectrogram (ADS) $[2,4]$ that is in turn used to extract feature set for target recognition without computing the ISAR image. The EAWT uses evolutionary programming for the T-F parameter extraction instead of FFT and the bisection search method used in the conventional AWT [5]. As all the parametric T-F analysis tools [2, 4-6] use parameter extraction as well as one or the other search methods while getting ISAR image, the computational complexity involved is quite high and hence hard to realize in real-time applications [6].

The cross-term problem inherent in the WVD degrades the quality of the ISAR image. In order to get better ISAR image, the problem of cross-term has to be reduced and is achieved with Choi-Williams distribution (CWD). The CWD reduces the cross-terms at the cost of time-frequency resolution, while still preserving the useful properties of the WVD. But this involves high computational complexity 
and is difficult to implement for practical scenarios. In [9], Newland modified the harmonic wavelets (HW) [10-12] for time-frequency representation (TFR), which is simple to implement compared to other wavelets and TFRs like WVD and CWD.

In order to trim down the computational complexity associated with ISAR imaging and make it viable for practical applications, the conception of TFR by HW is proposed in this paper for ISAR imaging. To capture the Doppler information effectively, high-frequency resolution is required, which is achieved with shorter frequency window function while computing the TFR by HW. The results from the simulated ISAR data show that the proposed method provides better image compared to that generated by CWD with reduction in computational complexity. Since the cost of the computational complexity plays an important role for practical scenarios, the proposed method is well suited for real-time implementation. The ISAR image thus obtained from the proposed method can be used for target identification using any of the existing methods. Here the neural network-based automatic target identification (ATI) scheme invariant to translation, rotation, and scale is used for identification and classification.

ATI is an important problem in the field of machine learning and pattern recognition. Hence, in the last two decades a large number of algorithms have been proposed. For example, Oja used the principle component analysis technique [13], Comon adopted the independent component approach [14], and Al-Ani et al. proposed a hybrid information algorithm [15] to deal with the problem of feature selection. Several methods were also proposed based on probability theory [16], fuzzy set theory [17], and artificial neural networks (ANNs) [18-20]. Further, the target recognition scheme discussed in [4] extracts the feature set directly from geometrical moments of the ADS without computing the ISAR image. But, the proposed method of recognition uses ISAR image for extracting the feature set. As the ISAR image gives the silhouette of the target, the trained operator can use his intelligence in addition to machine intelligence in classification and decision-making.

Any recognition process usually involves three components: preprocessing block, feature extractor, and classifier. The function of preprocessing block is to transform the input digital image into a form that can be processed and used by the subsequent blocks. Typical image-preprocessing functions are noise suppression, blur control, edge detection, and boundary extraction. In feature extractor, certain selective characteristics of the image are extracted that can uniquely distinguish the image from the other class of images. If the selected feature set is large, the preprocessing and analysis task becomes more difficult. On the other hand, if the feature set is small, the recognition rate may come down. Also, the extracted features should be invariant to certain parameters like scaling, shifting, and rotation depending on the scenario. As a result, feature selection has become important and wellknown problem. The classifier block compares these features with the feature set in the database according to a prede- fined similarity function and classifies the output image to one class of the stored images.

In this paper, region-growing technique is used for finding the centroid to overcome the problem of spurious edges and noise. A rotation invariant, translation invariant, and scale invariant feature set is selected for accurate classification [21]. Neural network-based classification is done instead of conventional template matching to increase the speed of matching and robustness to distorted patterns.

This paper is organized as follows. The basic of HW and its variation for ISAR imaging is discussed in Section 2. Neural network-based ATI using ISAR images invariant to translation, rotation, and scaling is discussed in Section 3. Section 4 presents simulated results for ISAR imaging and classification. Finally conclusions are made in Section 5.

\section{ISAR IMAGING USING TIME-FREQUENCY REPRESENTATION}

Radar transmits electromagnetic waves to a target and receives the reflected signal from the target. The received signal can be used to obtain the image of the target as it is related to the reflectivity function of the target by means of a filtering process. Figure 1 illustrates the process of the ISAR imaging system using a stepped-frequency (SF) waveform. For a stepped-frequency waveform, the radar transmits a sequence of $N$ bursts. Each burst consists of $M$ narrow-band pulses. Within each burst, the center frequency $f_{m}$ of each successive pulse is increased by a constant frequency step $\Delta f$. The total bandwidth of the burst, that is, $M$ times the frequency step $\Delta f$, determines the radar range resolution. The total number of bursts $N$ along with the pulse duration for a given imaging integration time determine the Doppler or cross-range resolution. The returned pulse is heterodyned and quadrature detected in the radar receiver.

To form a radar image, after measuring the returned inphase (I-Channel) and quadrature phase (Q-Channel) signals at baseband with a pulse repetition rate at $M * N$ time instants $t_{m, n}=(m+n M) \Delta t$, the $M \times N$ complex data is organized into a two-dimensional array which represents the unprocessed spatial frequency signature of the target $S\left(f_{m, n}\right)$, where $m=1,2, \ldots, M, n=1,2, \ldots, N$, and $\Delta t$ denotes the time interval between the pulses.

The radar processor uses the frequency signatures as the raw data to perform range compression and the standard motion compensation. Range compression functions as a matched filter, which removes frequency or phase modulation and resolves range. For the stepped-frequency signals, the range compression performs an $M$-point inverse FT (IFT) for each of the $N$ received frequency signatures as $G\left(r_{m, n}\right)=\operatorname{IFT}_{m}\left\{S\left(f_{m, n}\right)\right\}$, where $\operatorname{IFT}_{m}$ denotes the IFT operation with respect to the variable $m$. Therefore, $N$ range profiles (i.e., the distribution of the target reflectivity in range), each containing $M$ range cells, can be obtained. At each range cell, the $N$ range profiles constitute a new time history series. Then, the motion compensated range profiles become $G^{\prime}\left(r_{m, n}\right), m=1,2, \ldots, M, n=1,2, \ldots, N$. 

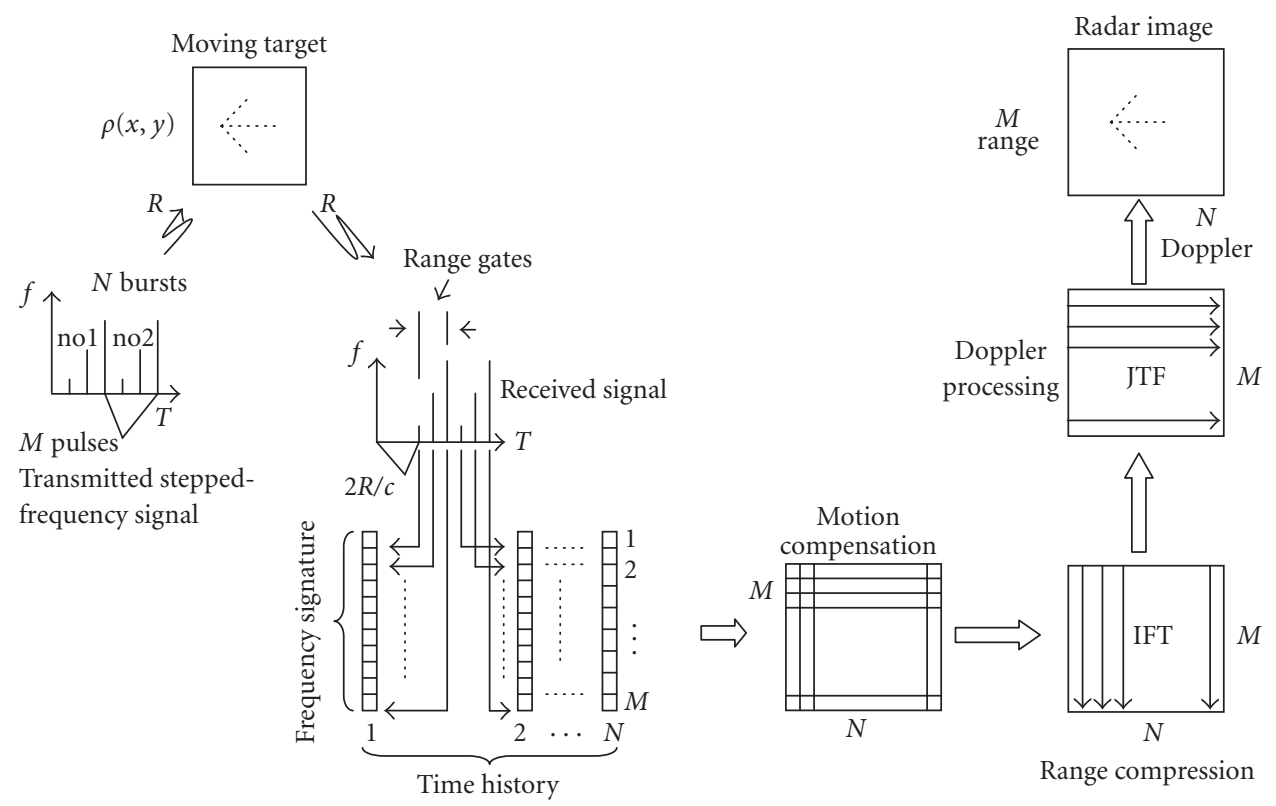

FIGURE 1: Illustration of SF radar imaging of moving target.

\subsection{Time-frequency representation}

TFR is an essential element in most of diagnostic signal analysis schemes. There is considerable interest in the effectiveness of different methods for generating TFRs, which describe the distribution of energy over frequency and time. The three main methods are: (1) the short-time Fourier transform (STFT), which generates a spectrogram, (2) the Wigner-Ville method of generating time-frequency distributions, and (3) the harmonic wavelet (HW) method of constructing wavelet maps, which is akin to TFR except that wavelet scale is plotted instead of frequency. All three methods generate a (real) function of time and frequency, which can be plotted to generate a surface on the time-frequency plane. For this purpose, wavelet scale is converted to frequency.

The Wigner-Ville distribution (WVD) is a TFR with excellent time and frequency resolution and several translation, modulation and marginal properties, and hence, is very useful for nonstationary signal analysis. The WVD of a signal $x(t)$ is given by $[7,8]$

$$
W_{x}(t, \omega)=\int_{-\infty}^{\infty} r(t, \tau) e^{-j \omega \tau} d \tau
$$

where $r(t, \tau)=x(t+\tau / 2) x^{*}(t-\tau / 2)$ is called the instantaneous autocorrelation function and the superscript $*$ indicates conjugate operation. Since the lag length $\tau$ can go to even infinity, the WVD theoretically can provide infinite frequency resolution. The WVD has two fundamental disadvantages: (1) computational complexity and (2) difficulty introduced by its spurious interference terms (cross-terms). The former is an important practical problem and the latter occurs when the signal contains more than one component because of the built-in quadratic nature of the WVD.
For real-time computations or for long-time series, this leads to inaccuracies and hence, it can be reduced by filtering the WVD with Choi-Williams kernel $e^{-\theta^{2} \tau^{2} / \sigma}$. This filtered WVD is also known as Choi-Williams distribution (CWD) as it uses Choi-Williams kernel to reduce the cross-terms and preserve the useful properties of the WVD with slightly reduced time-frequency resolution and largely reduced crossterm interference. The CWD of a signal $x(t)$ is given by $[7,8]$

$$
\begin{aligned}
& \operatorname{CWD}(t, \omega) \\
& \quad=\frac{1}{4 \pi^{3 / 2}} \iint \frac{1}{\sqrt{\tau^{2} / \sigma}} \exp \left[-\frac{(u-t)^{2}}{4 \tau^{2} / \sigma}-j \tau \omega\right] r(u, \tau) d u d \tau .
\end{aligned}
$$

For large values of $\sigma$, CWD approaches the WVD since the kernel approaches one and for small values of $\sigma$, the crossterm existing in WVD is reduced in CWD. But, this introduces extra computations.

\subsection{Harmonic wavelets $[9,10]$}

In essence, HW-based TFR is the same as the STFT except that any basis function can be used (only harmonic functions of constant amplitude and phase are used by the STFT). Usually the wavelets with a narrow frequency band are effective for time-frequency analysis; otherwise good frequency resolution is impossible. Subject to this narrow-band proviso, wavelets of any kind may be used for TFR, but HWs are particularly suitable because their spectrum is confined to a compact frequency band.

The input signal $x(t)$ is correlated with the basis function $w(t)$. Because $w(t)$ is localized and generally has harmonic characteristics, it is called a wavelet [9]. Any waveform may be used for the wavelet, provided that it must satisfy 


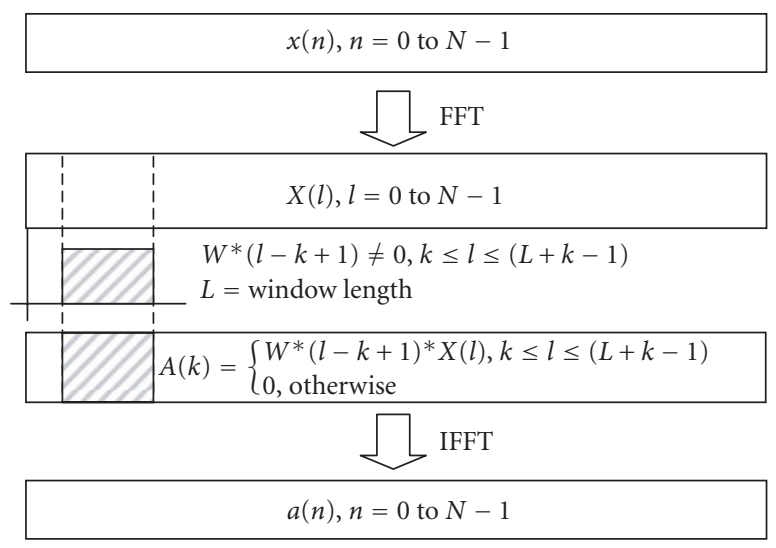

FIGURE 2: Schematic to compute harmonic wavelet coefficients.

admissibility and regularity conditions [22]. For an analyzing wavelet function $w(t)$, the wavelet transform coefficient $a(t)$ of a signal $x(t)$ is given by

$$
a(t)=\int_{-\infty}^{\infty} x(\tau) w(t+\tau) d \tau
$$

In terms of FT,

$$
\begin{aligned}
A(\omega) & =X(\omega) W^{*}(\omega), \\
a(t) & =F^{-1}\left[X(\omega) W^{*}(\omega)\right] .
\end{aligned}
$$

That is, the wavelet transform coefficients can be computed using inverse fast Fourier transform (IFFT) algorithm by (5) using $X(\omega)$ with $W(\omega)$ for different wavelet functions. Specifically, for the HW given by Newland $[10,11], W(\omega)$ is very simple and it is zero except over a finite band $[\pi / p, \pi / q]$, where $p, q$ can be real numbers, not necessarily integers. For $\mathrm{HW}$, the rectangular window $W(\omega)$, though compact in frequency domain, is of infinite duration in time domain. This can be overcome by using a proper smoother weighing function $W(\omega)$ other than a rectangular one.

A practical computation of HW for an input signal $x(t)$ sampled $N$ times is illustrated in Figure 2. In first stage, the FFT of the signal is computed. In second stage, the Fourier coefficients obtained are weighed by a frequency window function of length $L$ and the length of the resultant block is made equal to $N$ by padding $p$ leading zeros and $N-(L+p)$ trailing zeros. The IFFT of the resulting $N$ term series is then computed in third stage to determine the HW coefficients (HWCs) for that particular frequency band. Similar procedure is repeated for the successive frequency blocks by moving the frequency window along the frequency spectrum. It is shown in [9] that the number of added zeros both before and after the embedded block of Fourier coefficients can be changed while still preserving the HWCs. The data in the chosen frequency band is zero-padded to get smoothness over time, which can be further improved by multiplying the
FTs of a wider range of test functions, but data for equallyspaced times is always produced. Therefore, there is a duality between the STFT and HW method. The STFT produces results for local, short-time segments, covering the whole frequency spectrum in constant bandwidth steps whereas the HW method produces results for local, narrow frequency bandwidths, covering the whole duration of the record in constant time steps.

Both the STFT and WVD/CWD methods are constant bandwidth methods. Their algorithms require a transformation from the time domain to frequency domain by using the FT generating Fourier coefficients for frequencies at constant separation. The bandwidth of each frequency term is same. In contrast, the HW method allows the bandwidth of adjacent frequency terms to be chosen arbitrarily. Because the wavelet transform acts as a variable $\mathrm{Q}$ filter, where $\mathrm{Q}$ is the ratio of center frequency to bandwidth, it has greater flexibility than the other two methods. Further, the HW provides builtin decimation as well as interpolation if required $[11,12]$.

The fundamental advantage of the HW is that it offers a computationally efficient method for a variable bandwidth frequency transform so that the TFR can have a constant$\mathrm{Q}$ or a variable-Q basis as desired. In contrast, a TFR constructed by the STFT always has a constant bandwidth basis, therefore giving the same frequency resolution from low frequencies to high frequencies. Similar to STFT, the proposed method also suffers from tradeoff between time and frequency resolution. However, to capture the Doppler information effectively, better frequency resolution is required, which is achieved with shorter frequency window function while computing the HWCs.

\subsection{Harmonic wavelets for ISAR imaging}

In the proposed method, the HW-based TFR is customized for the purpose of ISAR imaging. Here all the stages of the HW method are similar but some extra step has to be followed before the second stage. That is, if length of the window used to truncate the spectrum of the signal is $L$ (assuming $L$ as even), then $L / 2$ zeros have to be padded before and after the spectrum of the signal so that total length of the modified spectrum is equal to the sum of lengths of the original spectrum and the window. If $L$ is odd, then $(L-1) / 2$ zeros have to be padded before and $(L+1) / 2$ zeros have to be padded after the spectrum. To capture the Doppler information, better frequency resolution is required, which is achieved by using a shorter window. As the window length is constant for different center frequencies, the TFR obtained by HW is of constant bandwidth just like that obtained by STFT and WVD/CWD.

The data consists of $N$ range profiles each containing $M$ range cells. The samples taken at the $i$ th range cell for the $N$ range profiles constitute a time history series. For the computation of $\operatorname{TFR}_{i}(n, k),(n=1,2, \ldots, N, k=1,2, \ldots, N)$, for $i$ th range cell, HW uses this time history series as an input to get

$$
\operatorname{TFR}_{i}(n, k)=\left|\operatorname{IFFT}\left[A_{k}(l)\right]\right|^{2}, \quad i=1,2, \ldots, M,
$$


where

$$
A_{k}(l)=\left\{\begin{aligned}
W(l-k+1) * X(l), & \\
\quad k \leq l \leq(L+k-1), & L: \text { window length } \\
0, & \text { otherwise. }
\end{aligned}\right.
$$

This procedure is repeated for each range cell $i$ to get $M$ number of TFRs. Finally, the ISAR image at $m$ th instant is obtained by

$$
I(m, k)=\left[\begin{array}{c}
\operatorname{TFR}_{1}(m, k) \\
\operatorname{TFR}_{2}(m, k) \\
\vdots \\
\operatorname{TFR}_{M}(m, k)
\end{array}\right], \quad k=1,2, \ldots, N
$$

Since $\operatorname{TFR}_{i}(m, k)$ captures the Doppler for every time instant, the image $I(m, k)$ obtained by $\operatorname{TFR}_{i}(m, k)$ through $(8)$ will be of better quality with reduced blurring.

\subsubsection{Algorithm for ISAR imaging by harmonic wavelets}

Step 1. The given data consists of $N$ range profiles each containing $M$ range cells. Compute the FT of $i$ th range cell by $X(l)=\operatorname{FFT}\left[x_{i}(n)\right]$, where $x_{i}(n)=x(i, n), n=1,2, \ldots, N$; $i=1,2, \ldots, M$, and $l$ is the discrete frequency bin index.

Step 2. Pad the equal number of zeros at the beginning and at the end of the spectrum of the signal such that the length of the modified spectrum is equal to the sum of lengths of the original spectrum $(N)$ and the window $(L)$, that is, $(N+L)$, therefore discrete frequency bin index $l=1,2, \ldots,(N+L)$. This is to preserve the spectral information that may be lost otherwise.

Step 3. Compute the TFR of $i$ th range cell using HW. For this:

(i) compute the weighted Fourier coefficients at the $k$ th discrete frequency index using

$$
A_{k}(l)= \begin{cases}W(l-k+1) * X(l), & k \leq l \leq(L+k-1) \\ 0, & \text { otherwise }\end{cases}
$$

where $W(p)$ is the window of length $L, p=1,2, \ldots, L$, (ii) the HWCs $a_{k}(n)$ are computed by taking IFFT of $A_{k}(l)$,

(iii) squared magnitudes of the HWCs are computed by $\operatorname{TFR}_{i}(n, k)=\left|a_{k}(n)\right|^{2}$,

(iv) repeat steps (i), (ii), (iii) for different frequency indices $k=1,2, \ldots, N$ to get the complete TFR of the $i$ th range cell.

Step 4. Repeat steps $1,2,3$ to get $\operatorname{TFR}_{i}(n, k)$ for different range cells $i=1,2, \ldots, M$.
TABLE 1: Computational complexity by different methods.

\begin{tabular}{l|c|c}
\hline & $\begin{array}{c}\text { Number of } \\
\text { multiplications }\end{array}$ & $\begin{array}{c}\text { Number of } \\
\text { additions }\end{array}$ \\
\hline STFT & $3276800=32.768 * 10^{5}$ & $3145728=31.45728 * 10^{5}$ \\
CWD & $44058624=440.58624 * 10^{5}$ & $44040192=440.40192 * 10^{5}$ \\
HW & $3456636=34.56636 * 10^{5}$ & $3440252=34.40252 * 10^{5}$ \\
\hline
\end{tabular}

Step 5. The range-Doppler image frame at $n$th time instant is obtained by combining the respective $n$th Doppler spectrum from each of $\operatorname{TFR}_{i}(n, k)$ for $i=1,2, \ldots, M$.

Steps 1 to 5 form the algorithm for ISAR imaging by HW.

\subsubsection{Computational complexity}

To compare the computational complexity of ISAR imaging by STFT, CWD, and HW, the data of $N$ range profiles each with $M$ range cells is considered. The computation of a single TFR by STFT requires " $N$ " $N$-point FFTs for each time history. Hence the computation of " $M$ " TFRs for each time history requires " $(N * M)$ " $N$-point FFTs. Further, the use of any window of length $L_{s}$ requires $\left(N * L_{s}\right)$ multiplications for the computation of a single TFR and thus the computation of " $M$ " TFRs requires $\left[\left(N * L_{s}\right) * M\right]$ multiplications. From these " $M$ " TFRs, " $M$ " ISAR images can be obtained.

The computation of a single TFR by CWD involves $\left(N_{w}^{2} / 8\right)$ multiplications (to compute instantaneous autocorrelation function), " $N_{w}$ " $N_{w}$-point IFFTs (to compute ambiguity function), $\left(N_{w} * N_{w}\right)$ multiplications (for cross-term reduction by windowing), and " $\left(2 * N_{w}\right)$ ” $N_{w}$-point FFTs (2$D$ FFT of size $N_{w} \times N_{w}$ ), where $N_{w}=2 * N$. Accordingly, the computation of " $M$ " TFRs needs $M$ times the above computations, that is, $\left[\left(N_{w}^{2} / 8\right)+\left(N_{w} * N_{w}\right)\right] * M$ multiplications and $\left[N_{w}+\left(2 * N_{w}\right)\right] * M$ number of $N_{w}$-point FFTs.

On the other hand, the computation of a single TFR by HW requires one $N$-point FFT and " $N$ " $(N+L)$-point IFFTs for each time history. Also, the use of window of length $L$ for the computation of a single TFR requires $(N * L)$ multiplications. Consequently, the computation of " $M$ " ISAR images requires $[(N * L) * M]$ multiplications, " $M$ " $N$-point FFTs, and " $(N * M)$ " $(N+L)$-point FFTs.

As the FFT lengths are different for different methods, the computational complexities involved in the methods are compared in terms of multiplications and additions. For this, the computation of a single $N$-point FFT requires $2 * N *$ $\log _{2}(N)$ real multiplications and $2 * N * \log _{2}(N)$ real additions. Table 1 shows the computations required by different methods in terms of multiplications and additions for the following parameters: $N=64, M=64, L=4, L_{s}=32$, and $N_{w}=2 * N=128$.

From Table 1, it is evident that, even though the ISAR image by HW has increased the computational complexity by $5.4882 \%$ in terms of multiplications and $9.3627 \%$ in terms of additions compared to STFT, it has reduced the computational complexity by $92.1545 \%$ in terms of multiplications and $92.18 \%$ in terms of additions compared to CWD. Hence, 


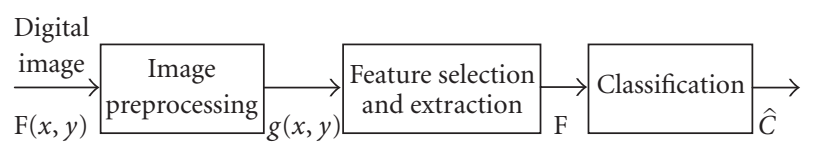

Figure 3: Typical image pattern recognition system.

the proposed method is better suited for practical scenarios with reduction in computations while maintaining similar/better results compared to CWD.

\section{TARGET RECOGNITION}

A typical pattern recognition process usually involves three components, preprocessing block, feature extractor, and a classifier. Figure 3 shows the block diagram of a typical image pattern recognition system. Input to the system is a digital image. However, this image may not be in a state that can be processed. The function of image preprocessing block is to transform this input digital image $f(x, y)$ into a form $g(x, y)$ that can be processed and used by the subsequent blocks. Typical image-preprocessing functions required are noise suppression, blurring control, and edge detection.

In most cases, entire image may not be required for carrying out the pattern recognition process. Certain selective characteristics of the image can retain the uniqueness of the image. Such characteristics are called primitive features. These primitive features are to be extracted from the preprocessed image. Further, a typical recognition system needs to recognize only few classes of objects. Hence, among the primitive features, only certain features of the image (F) can uniquely distinguish it from the other classes of image. These features are identified and selected by the feature extraction block of the system shown in Figure 3. The classifier block then compares these features with the features of the image in its database according to a predefined "similarity" function and recognizes the output image.

ATI using ISAR images is a challenging task because of low SNR, poor resolution, and blur associated with the ISAR images. So preprocessing block is essential before feature extraction and classification. Median filtering [23] is used for removing the point-spread noise. Unwanted patches are removed and the object is extracted using the standard region-growing technique [24]. After the object is extracted from background with region-growing method, all the pixel positions within the region of interest (ROI) are well known. Giving equal importance to all the pixels within ROI, centroid calculation reduces to simple average of all horizontal and vertical positions of the pixels within ROI. Features are extracted from the test patterns by applying FFT and wavelet transforms to the polar-transformed original patterns. Finally classification is done using the neural networks.

\subsection{Feature selection and extraction}

For feature selection, both Fourier descriptors and wavelet descriptors are considered. Fourier descriptor has been a powerful tool for recognition because it has a useful property of shift invariance with respect to spectrum. However, the frequency information obtained from the Fourier descriptor is global, a local variation of the shape can affect all Fourier coefficients. In addition, Fourier descriptor does not have a multiresolution representation. On the other hand, wavelet descriptors have multiresolution property, but they are not translation invariant. A small shift of original signal will cause totally different wavelet coefficients. Since Fourier descriptor and wavelet descriptor both have good properties and drawbacks, they are combined to obtain the descriptor, which is invariant to translation, rotation, and scaling.

Feature extraction is a crucial processing step for pattern recognition. Some methods extract 1-D features from 2-D patterns. The advantage of this approach is that space can be saved for the database and the time for matching through the whole database can be reduced. The apparent drawback is that the recognition rate may not be very high because less information from the original pattern is retained. In this paper, 2-D features for pattern recognition is used in order to achieve higher recognition rate [25].

The translation invariance is achieved by translating the origin of the coordinate system to the center of the image pattern or object, denoted by $\left(x_{0}, y_{0}\right)$. As the center of the object is considered as the reference point for the next level processing, the recognition scheme is invariant to translation of the object within the frame.

The scale invariance is obtained by transforming the image pattern $f(x, y)$ into normalized polar coordinate system. Let

$$
d=\max _{f(x, y) \neq 0} \sqrt{\left(x-x_{0}\right)^{2}+\left(y-y_{0}\right)^{2}}
$$

be the longest distance from $\left(x_{0}, y_{0}\right)$ to a point $(x, y)$ on the pattern. $N$ concentric circles are drawn centered at $\left(x_{0}, y_{0}\right)$ with radius $d * I / N, I=1,2,3 \ldots, N$. Also, $N$ angularly equal-spaced radial vectors $\theta_{j}$ departing from $\left(x_{0}, y_{0}\right)$ with angular step $2 \pi / N$ are drawn. For any small region,

$$
\begin{gathered}
S_{i, j}=\left\{(r, \theta) \mid r_{i}<r \leq r_{i+1}, \theta_{j}<\theta \leq \theta_{j+1}\right\}, \\
i=0,1, \ldots,(N-1), j=0,1, \ldots,(N-1)
\end{gathered}
$$

calculate the average value of $f(x, y)$ over this region, and assign the average value to $g(r, \theta)$ in the polar coordinate system. The feature $g(r, \theta)$ obtained in this way is invariant to scaling, but the rows may be circularly shifted if we use different orientation.

With regard to rotational invariance, 1-D FT is applied along the axis of polar angle $\theta$ of $g(r, \theta)$ to obtain its spectrum. Since the spectra of FT of circular shifted signals are the same, we obtain a feature, which is rotation invariant. Multiresolution feature of wavelet is used to get a compact feature set, which in turn reduces computational complexity and memory requirements. Different wavelet families like Haar, Bior, and Daubechies are considered. Haar wavelet is chosen as other wavelets do not improve the recognition rate much and are computationally more intensive. Haar wavelet transform is applied along the range axis to obtain the finer 


\begin{tabular}{|c|c|c|c|c|c|c|}
\hline$f(x, y)$ & Polarize & $g(r, \theta$ & 1-D FFT & $\begin{array}{c}G(r, \Phi)= \\
\mathrm{FT}(\sigma(r, \theta))\end{array}$ & 1-D WT & $\mathrm{WT}_{r}(G(r, \phi))$ \\
\hline
\end{tabular}

FIGURE 4: Block diagram of feature extraction algorithm.

level feature set. Lifting scheme is used for implementing the Haar wavelet transform because of its less computational complexity and memory requirements.

\subsubsection{Feature extraction algorithm}

The steps of the algorithm can be summarized as follows (also shown in Figure 4):

(1) find the centroid of the pattern $f(x, y)$ and transform $f(x, y)$ into polar coordinate system to obtain $g(r, \theta)$,

(2) conduct 1-D FT on $g(r, \theta)$ along the axis of polar angle $\theta$ and obtain its spectrum,

(3) apply 1-D wavelet transform on $G(r, \Phi)$ along the axis of radius $r$.

\subsection{Neural network-based pattern recognition}

Back propagation network is an ideal choice to serve as a pattern classifier because it has been used successfully in various pattern recognition applications with good recognition results. In the back propagation algorithm, the information about errors at the output units is propagated back to the hidden units. The number of input units depends on the size of feature vector. The training of a network by back propagation involves three stages: the feed forward of the input training pattern, the calculation and back propagation of the associated error, and the adjustment of the weights. Through a set of learning samples, the network can find the best weights $W_{i j}$ automatically, enabling it to exhibit optimal classification ability. After training, application of the net involves only computations of the feed forward phase. Even if training is slow, a trained net can produce its output very rapidly.

Feature vectors coming from feature selection and extraction block are given as input to the neural network. Back propagation network with one input layer, one hidden layer, and one output layer is used for classification. The activation function used is a binary sigmoidal function, which has a range of $(0,1)$ and is defined as

$$
\begin{aligned}
f(x) & =\frac{1}{1+e^{-x}}, \\
f^{\prime}(x) & =f(x)(1-f(x)) .
\end{aligned}
$$

The initial weights are set at random. In the training process, weights are updated in such a way as to minimize the mean square difference between the actual and desired output. Finally the pattern is classified according to the output sequence of the neural network.

\section{SIMULATION RESULTS}

The radar data used for simulation is obtained from the stepped-frequency radar operating at $9 \mathrm{GHz}$ and has a bandwidth of $512 \mathrm{MHz}$ (for MIG-25), $150 \mathrm{MHz}$ (for B-727) [http://airborne.nrl.navy.mil/ vchen/tftsa.html]. The radar data of MIG-25 consists of 512 successive pulses with each pulse having 64 complex range samples and that of B-727 consists of 256 successive pulses with each pulse having 64 complex range samples.

The performance of the proposed method of ISAR imaging is compared with the existing methods using FT, STFT, and CWD and is illustrated for both the aircrafts. Figures 5 and 6 illustrate the performance comparison of the proposed method for MIG-25. Use of FT for ISAR imaging assumes that Doppler frequency is constant over the imaging time duration. However, the assumption of constant Doppler frequency is not true as the Doppler frequency varies in time because of the nonuniform motion of the target. Hence the ISAR image computed by FT often leads to blurring, which is illustrated in Figure 5(a). The ISAR images (frame 30) obtained by STFT, CWD, and HW are shown in Figures 5(b), (c), and (d), respectively. It is observed that the ISAR image obtained by CWD (Figure 5(c)) is prolonged in Doppler frequency. This is because the spectral peaks will occur at twice the desired frequencies due to built-in nature of the WVD. Even though the proposed method requires one extra FT (to compute the spectrum of the signal for a single TFR) compared to STFT, it provides better Doppler frequency resolution. Further, ISAR image by HW provides better Doppler frequency resolution compared to CWD, with reduced computations.

The blurred ISAR image obtained by FT of frame-1 is shown in Figure 6(a). The frame-1 images of MIG-25 obtained by CWD and STFT are of poor quality compared to that obtained by HW (Figure 6). That is, the proposed method gives better image quality compared to other nonparametric methods with reduced computations. Similar results are shown in Figure 7 (frame-30) and Figure 8 (frame-1) for B-727 to compare the performance of the proposed method with the existing methods. Because of the complex motion of the target B-727, the image by FT suffers from blurring (Figures 7(a), 8(a)), which is not observed in other methods (Figures 7(b), (c), (d)). Also, it is observed that images obtained by STFT and CWD do not show the wings, wingtips, and tail of the target clearly (Figures 7(b), (c), 8(b), (c)), but are visible to some extent with HW (Figures $7(\mathrm{~d}), 8(\mathrm{~d})$ ). Further, the proposed method provides better and consistent results for all the frames compared to other two methods with reduced computational complexity.

For the computation of ISAR image by CWD, $\sigma$ is chosen to be 0.05 to reduce the cross-terms as much as possible. In all the cases for the computation of ISAR image by STFT, a rectangular window is used as it provides better frequency resolution compared with other windows. However, the STFT suffers from tradeoff between time resolution and frequency (Doppler frequency) resolution depending on the length of the window chosen. The STFT is used for computing the 


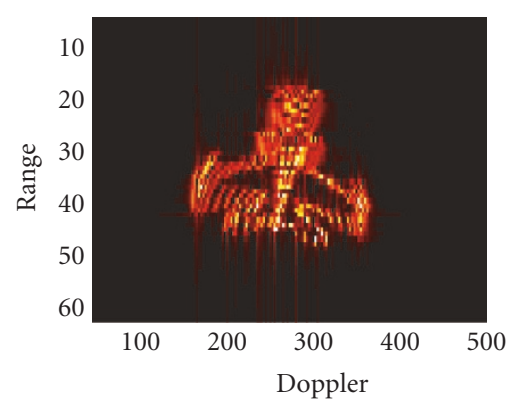

(a)

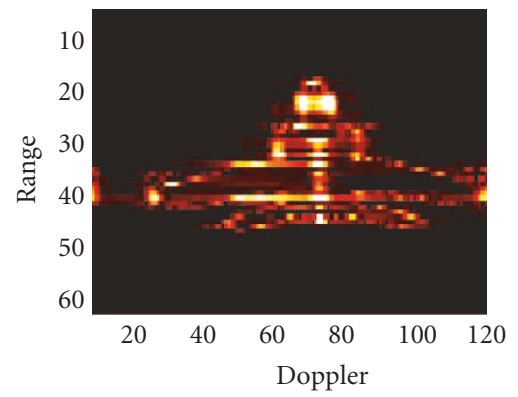

(c)

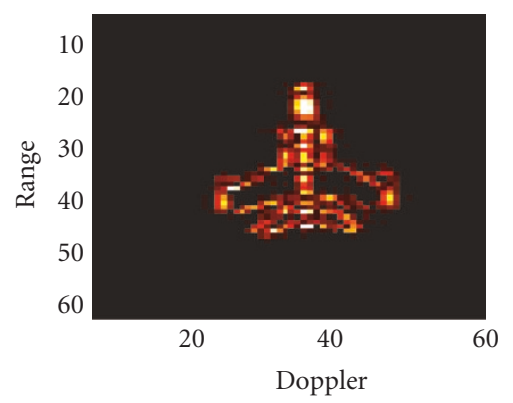

(b)

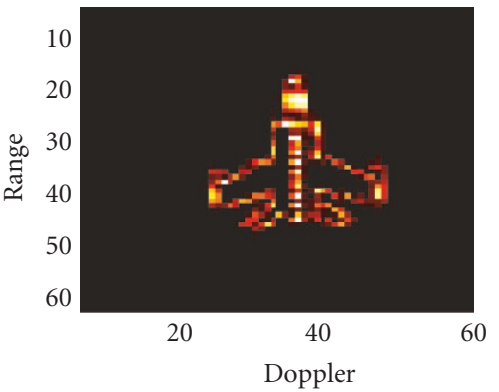

(d)

Figure 5: Images of simulated MIG-25 by (a) FT, (b) STFT (frame-30), (c) CWD (frame-30), (d) HW (frame-30).

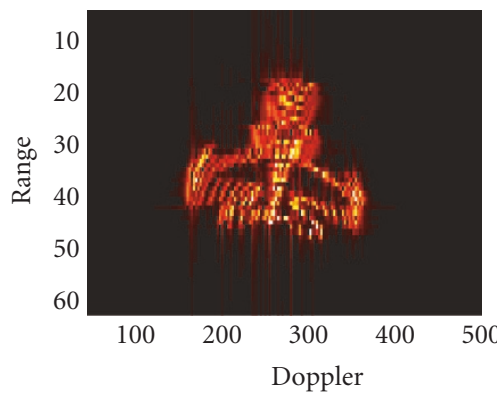

(a)

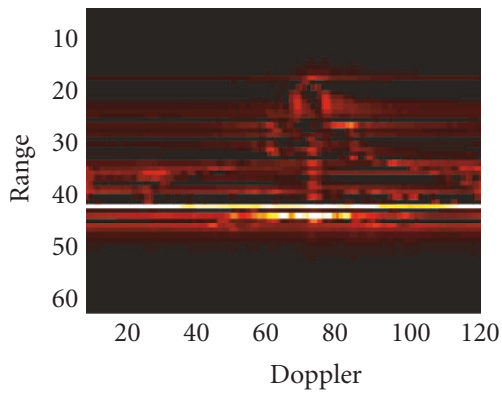

(c)

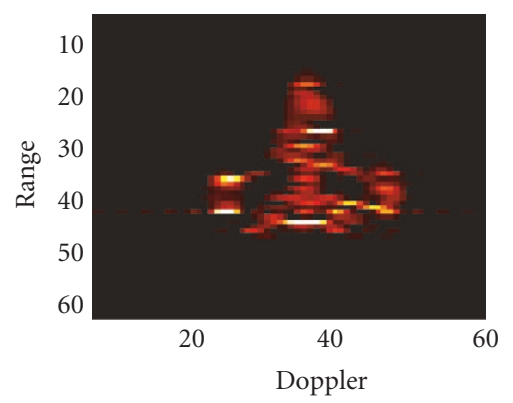

(b)

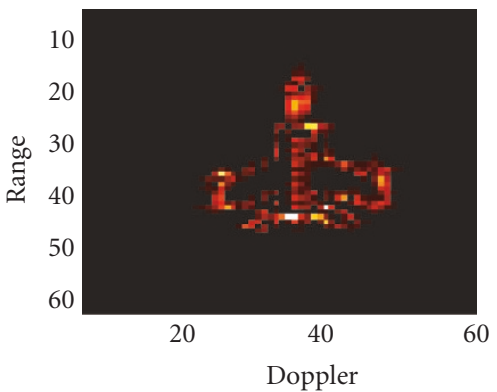

(d)

FIgURE 6: Images of simulated MIG-25 by (a) FT, (b) STFT (frame-1), (c) CWD (frame-1), (d) HW (frame-1). 


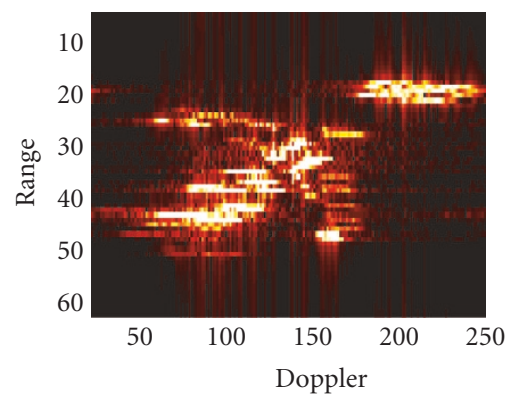

(a)

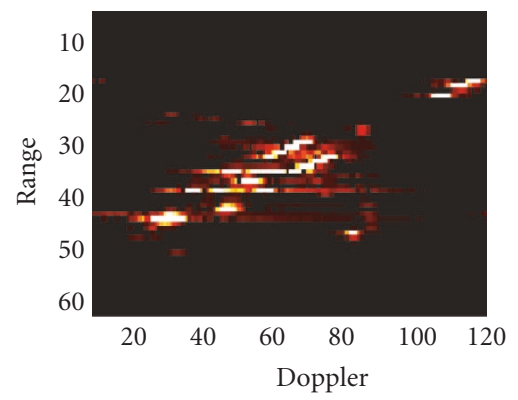

(c)

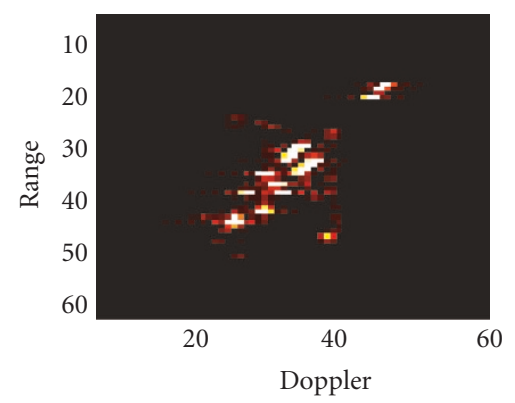

(b)

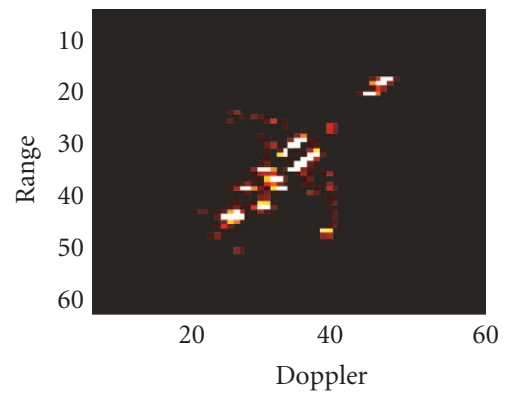

(d)

FIGURE 7: Images of simulated B-727 by (a) FT, (b) STFT (frame-30), (c) CWD (frame-30), (d) HW (frame-30).

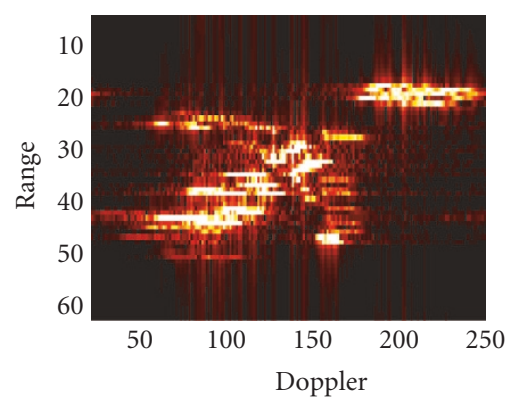

(a)

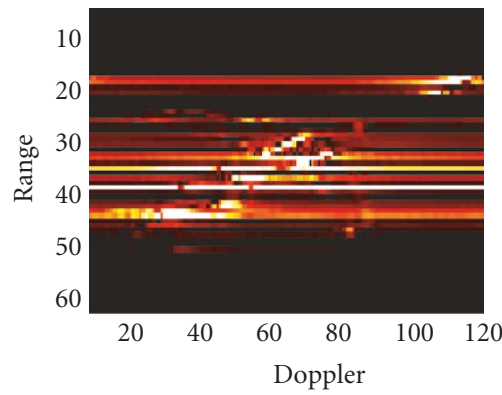

(c)

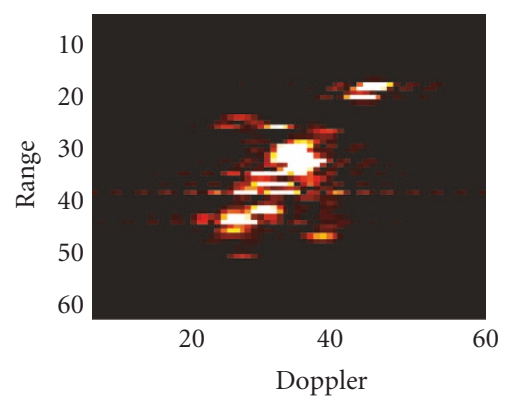

(b)

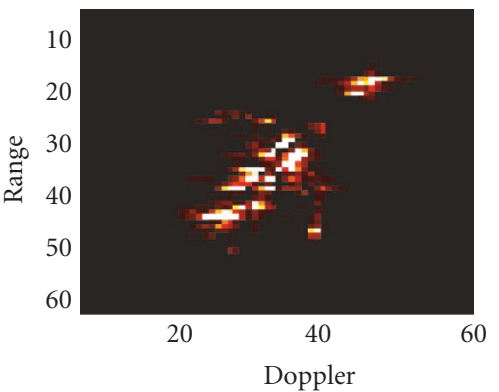

(d)

Figure 8: Images of simulated B-727 by (a) FT, (b) STFT (frame-1), (c) CWD (frame-1), (d) HW (frame-1). 


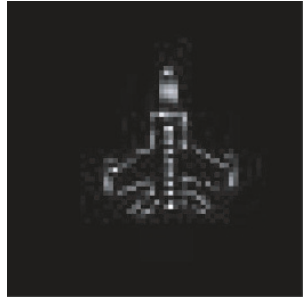

(a) ISAR image.

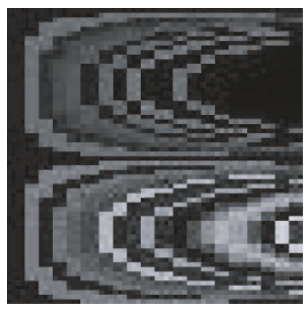

(c) Polar transform.

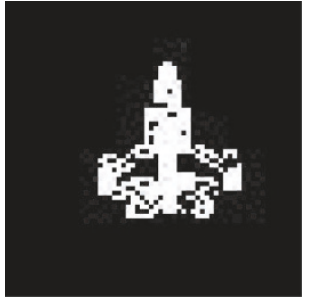

(b) Region growing.

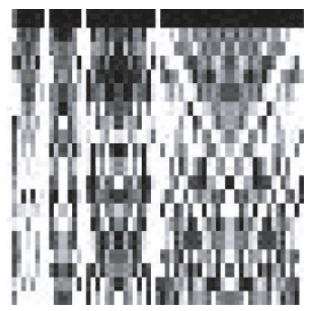

(d) Lifting.
FIgUre 9: Simulation results for MIG-25.

ISAR image with the assumption that the target motion is uniform within the window duration. But this may not be true for longer window duration and hence degrades the ISAR image. Further, to capture the Doppler information effectively, better frequency resolution is required, which is achieved with longer window provided that the data available is sufficient. If sufficient amount of data is not available, then the window length should be chosen such that it provides better frequency resolution. Here, the window length of 32 is used for the computation of STFT as it provides better results. Like STFT, TFR by HW also suffers from tradeoff between time resolution and frequency resolution. That is, the shorter the window length, the better the frequency resolution with poorer time resolution would be, and vice versa. This is because TFR by HW involves windowing the spectrum instead of data as in STFT. As better frequency resolution is required to capture the Doppler information effectively, shorter window is considered while computing the TFR by HW. Further, use of rectangular window generates $\mathrm{HW}$ of infinite duration in time. Use of a proper smoothing window other than a rectangular one makes it finite in time. Considering the above arguments, hamming window of length 4 for computing the TFR by HW is found to provide better results.

The reconstructed gray scale ISAR images of size $64 \times 64$ are given as input to the target recognition block. The results are shown in Figures 9, 10, 11, and 12 for different image patterns. The efficiency of the region-growing technique over edge-based technique in object extraction can be seen. The polar pattern for the rotated MIG-25 (Figure 10) and MIG-25 (Figure 9) can be compared to visualize how the polar transform converts object rotation into circular shifts for applying the FT. Wavelet transform is applied along each row (range) to get the finer level coefficients, which will help in minimizing the feature set size and thereby memory re-

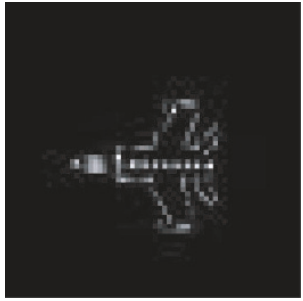

(a) ISAR image.

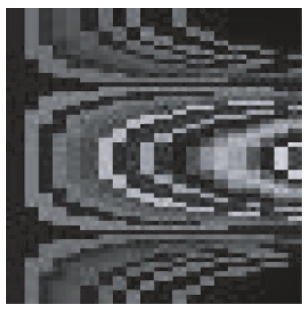

(c) Polar transform.

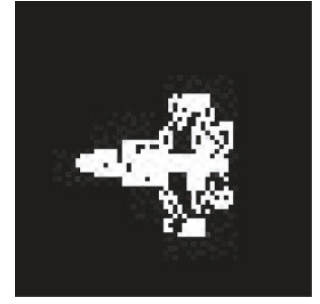

(b) Region growing.

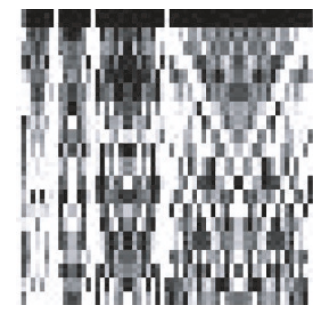

(d) Lifting.
FIgure 10: Simulation results for rotated MIG-25.

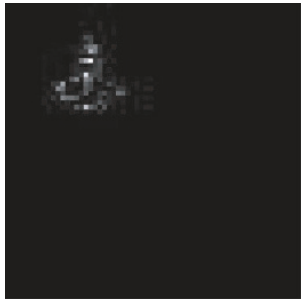

(a) ISAR image.

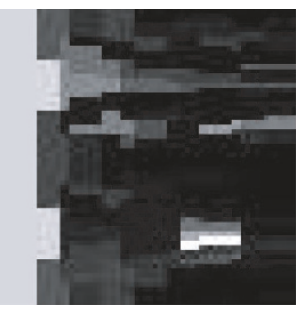

(c) Polar transform.

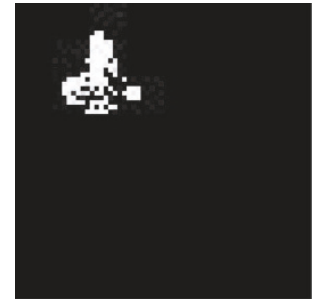

(b) Region growing.

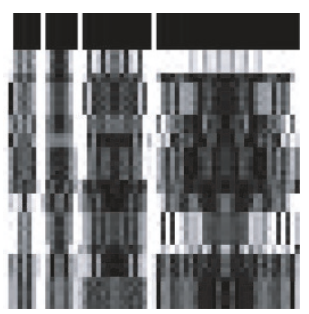

(d) Lifting.
FIGURE 11: Simulation results for 50\% scaled MIG-25.

quirements. Further, finer level wavelet coefficients with the decimated Fourier coefficients $(8 \times 8)$ are taken as the feature set for classification. Back propagation network with input layer of 64 nodes, one hidden layer of 32 nodes, and output layer of 9 nodes is used for classification.

\subsection{Classification results}

For classification, a set of 8 images in each category with 9 such ISAR image categories is considered. The system is trained with three images from each category and tested on remaining 5 images outside the training data. Classification 


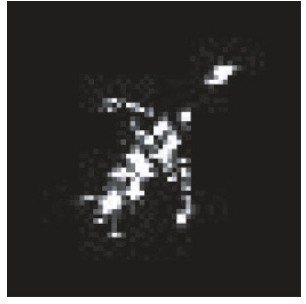

(a) ISAR image.

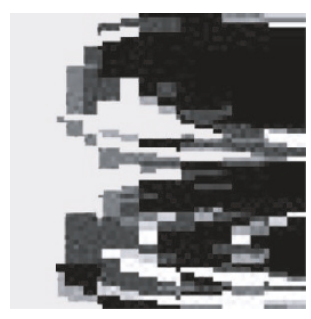

(c) Polar transform.

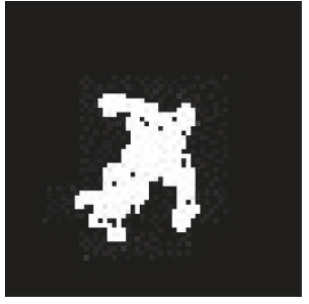

(b) Region growing.

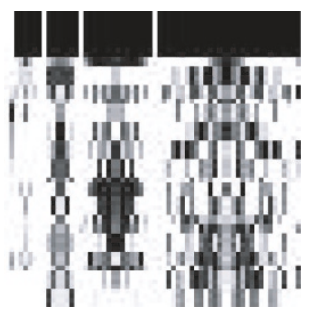

(d) Lifting.
FIGURE 12: Simulation results for B-727.

TABLE 2: Recognition results for different scaling factors.

\begin{tabular}{l|rrcrrr}
\hline \multirow{2}{*}{ Percentage (\%) } & \multicolumn{7}{|c}{ Scaling factor } \\
& \multicolumn{1}{|c}{0.5} & \multicolumn{1}{c}{0.6} & 0.7 & 0.8 & 0.9 & 1.2 \\
\hline Recognition rate & 93.73 & 97.38 & 98.68 & 100 & 100 & 100 \\
Error rate & 2.12 & 0.27 & 0 & 0 & 0 & 0 \\
Rejection rate & 4.15 & 2.35 & 1.32 & 0 & 0 & 0 \\
\hline
\end{tabular}

results for different scaling factors are shown in Table 2. Recognition rate is defined as number of targets properly identified per total number of targets, error rate is defined as number of targets falsely identified per total number of targets, and rejection rate is defined as number of unknown targets per total number of targets. Classification results for different rotation angles are not given, as the change in recognition results is nominal. As the scaling factor decreases, the extent of interpolation required in getting the feature set increases, thereby decreasing the recognition rate.

\section{CONCLUSIONS}

A new method of ISAR imaging based on TFR by HW is proposed in order to reduce the computations involved in ISAR imaging. The performance of the proposed method is compared with CWD as well as STFT in terms of performance as well as computational complexity. Further, the proposed method provides similar/better results with significant reduction $(92 \%)$ in computations compared to that by CWD. The ISAR images obtained from the proposed method are used for target identification. For target identification, a neural network-based ATI scheme is used which is invariant to translation, rotation, and scaling. Because of low SNR and poor resolution of ISAR images, region-growing technique is used instead of conventional edge-based techniques to improve the accuracy in centroid calculation. The use of feature set that is invariant to translation, rotation, and scaling, achieves good recognition results for all test patterns.

\section{Abbrevations}

ISAR Inverse synthetic aperture radar

$\mathrm{RF}$

Radio frequency

LFM Linear frequency modulation

SFM Stepped-frequency modulation

HW Harmonic wavelet

FT Fourier transform

IFT Inverse Fourier transform

STFT Short-time Fourier transform

FFT Fast Fourier transform

WVD Wigner-Ville distribution

CWD Choi-Williams distribution

TFR Time-frequency resolution

ANN Artificial neural networks

HWC Harmonic wavelet coefficients

CWT Continuous wavelet transform

AJTFT Adaptive joint time-frequency transform

AWT Adaptive wavelet transform

EAWT Evolutionary adaptive wavelet transform

ADS

Adaptive spectrogram

ATI Automatic target identification

\section{ACKNOWLEDGMENTS}

The first author is grateful to Dr. S. V. Narasimhan, Deputy Director, DSP Systems Lab, National Aerospace Laboratories, Bangalore, India, who taught him harmonic wavelets. The authors would like to express their gratitude to the anonymous reviewers for their helpful and constructive comments. The authors are thankful to Mr. C. R. Patil, Member (Research Staff), Central Research Laboratory, Bangalore, India, for reviewing and helpful comments.

\section{REFERENCES}

[1] V. C. Chen and S. Qian, "Joint time-frequency transform for radar range-Doppler imaging," IEEE Transactions on Aerospace and Electronic Systems, vol. 34, no. 2, pp. 486-499, 1998.

[2] V. C. Chen and H. Ling, Time-Frequency Transforms for Radar Imaging and Signal Analysis, Artech House, Boston, Mass, USA, 2002.

[3] H. Kim and H. Ling, "Wavelet analysis of radar echo from finite-size targets," IEEE Transactions on Antennas and Propagation, vol. 41, no. 2, pp. 200-207, 1993. 
[4] K.-T. Kim, I.-S. Choi, and H.-T. Kim, "Efficient radar target classification using adaptive joint time-frequency processing," IEEE Transactions on Antennas and Propagation, vol. 48, no. 12, pp. 1789-1801, 2000.

[5] V. C. Chen, "Reconstruction of inverse synthetic aperture radar image using adaptive time-frequency wavelet transform," in Wavelet Applications II, vol. 2491 of Proceedings of SPIE, pp. 373-386, Orlando, Fla, USA, April 1995.

[6] I.-S. Choi, B.-L. Cho, and H.-T. Kim, "ISAR motion compensation using evolutionary adaptive wavelet transform," IEE Proceedings of Radar, Sonar and Navigation, vol. 150, no. 4, pp. 229-233, 2003.

[7] L. Cohen, Time-Frequency Analysis, Prentice-Hall, Englewood Cliffs, NJ, USA, 1995.

[8] L. Cohen, "Time-frequency distributions - a review," Proceedings of the IEEE, vol. 77, no. 7, pp. 941-981, 1989.

[9] D. E. Newland, "Practical signal analysis: do wavelets make any difference?" in Proceedings of Design Engineering Technical Conferences (DETC '97), Sacramento, Calif, USA, September 1997.

[10] D. E. Newland, Random Vibrations, Spectral and Wavelets Analysis, Longman, Singapore, 3rd edition, 1993.

[11] D. E. Newland, "Wavelet analysis of vibration, part I: theory," Transactions of the ASME: Journal of Vibration and Acoustics, vol. 116, no. 4, pp. 409-416, 1994.

[12] D. E. Newland, "Time-frequency and time-scale signal analysis by harmonic wavelets," in Proceedings of the 1st European Conference on Signal Analysis and Prediction, pp. 53-59, Prague, Czech Republic, June 1997.

[13] E. Oja, "Neural networks, principal components, and subspaces," International Journal of Neural Systems, vol. 1, no. 1, pp. 61-68, 1989.

[14] P. Comon, "Independent component analysis, a new concept?” Signal Processing, vol. 36, no. 3, pp. 287-314, 1994.

[15] A. Al-Ani and M. Deriche, "A hybrid information maximisation (HIM) algorithm for optimal feature selection from multi-channel data," in Proceedings of IEEE International Conference on Acoustics, Speech, and Signal Processing (ICASSP '00), vol. 6, pp. 3470-3473, Istanbul, Turkey, June 2000.

[16] P. A. Devijver and J. Kittler, Pattern Recognition: A Statistical Approach, Prentice-Hall, London, UK, 1982.

[17] S. K. Pal, "Fuzzy set theoretic measures for automatic feature evaluation: II," Information Sciences, vol. 64, no. 1-2, pp. 165179, 1992.

[18] R. Battiti, "Using mutual information for selecting features in supervised neural net learning," IEEE Transactions on Neural Networks, vol. 5, no. 4, pp. 537-550, 1994.

[19] L. M. Belue and K. W. Bauer Jr., "Determining input features for multilayer perceptrons," Neurocomputing, vol. 7, no. 2, pp. 111-121, 1995.

[20] D. W. Ruck, S. K. Rogers, and M. Kabrisky, "Feature selection using a multilayer perceptron," Journal of Neural Network Computing, vol. 2, no. 2, pp. 40-48, 1990.

[21] P. Wunsch and A. F. Laine, "Wavelet descriptors for multiresolution recognition of handprinted characters," Pattern Recognition, vol. 28, no. 8, pp. 1237-1249, 1995.

[22] I. Daubechies, Ten Lectures on Wavelets, vol. 61 of CBMSNSF Regional Conference Series in Applied Mathematics, SIAM, Philadelphia, Pa, USA, 2nd edition, 1992.
[23] R. C. Gonzalez and R. E. Woods, Digital Image Processing, Addison Wesley, Boston, Mass, USA, 1993.

[24] Y. Yokoyama, Y. Miyamoto, and M. Ohta, "Very low bit rate video coding using arbitrarily shaped region-based motion compensation," IEEE Transactions on Circuits and Systems for Video Technology, vol. 5, no. 6, pp. 500-507, 1995.

[25] G. H. Granulund, "Fourier processing for handwritten character recognition," IEEE Transactions on Computers, vol. 21, pp. 195-201, 1992.

B. K. Shreyamsha Kumar received the B.E. degree in electronics and communication engineering from Bangalore University, India, in 2000. He received the M.Tech. degree in industrial electronics from National Institute of Technology, Karnataka, Surathkal, India, in 2004. He joined Central Research Laboratory as a Member (Research Staff) in 2004. He has two international conference papers in to his credit. His areas of research

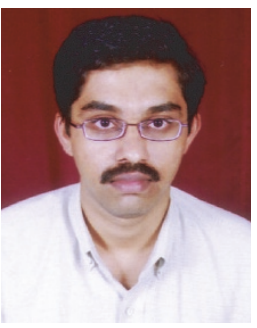
include group delay functions, spectral estimation, time-frequency representation, inverse synthetic aperture radar imaging, wavelets and image processing.

B. Prabhakar received the B.Tech. degree in electronics and communication engineering from Shri Venkateswara University, Tirupati, India, in 1997, and continued as a Faculty Member for one year. He received the M.Tech degree in digital signal processing from Indian Institute of Technology, Kanpur, India, in 2000. He joined Central Research Laboratory as a Member (Research Staff) in 2000. He has more than 10 research papers in international as well as national conferences. His research activities include image compression, video compression, tracking and pattern recognition.

K. Suryanarayana received the B.Tech. degree from Mangalore University, India, in 1998. He received the M.Tech. degree in system analysis and computer application from Karnataka Regional Engineering College, Surathkal, India, in 2001. He joined Central Research Laboratory as a Member (Research Staff) in 2001. He has more than five research papers in international as well as national conferences. His research activities include radar imaging, multitarget tracking and radar signal processing.

V. Thilagavathi received B.E. degree in electronic and communication engineering from VMKVEC, Salem, India, in 1996 and M.E. degree in applied electronics from PSG Institute of Technology, Coimbatore, India, in 2002. She has been working in Central Research Laboratory since 2002. She has three research papers in international as well as national conferences. Her research activities are image compression, video coding, video communication, and pattern recognition.
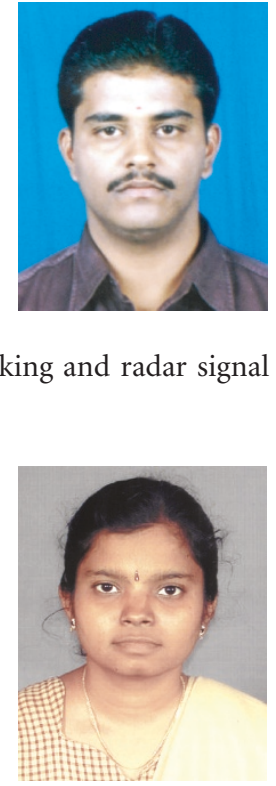
R. Rajagopal graduated from Madras University with B.E. (Honors) in 1982. He got his M.E. and Ph.D. degrees from Bharathidasan University, Tiruchirappalli. He worked in the Regional Engineering College, Trichy, in various capacities from 1982-1998. He joined Central Research Laboratory, Bangalore, in June 1998. He is currently Head of Radar Signal Processing

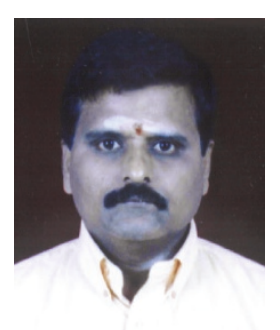
Group. He has published more than 50 re-

search papers in international journals and proceedings of international conferences. He is listed in Who's Who in the World, Who's Who in Science and Technology, Who's Who in Finance and Industry. He received the R\&D Award in 2003 for his contribution in the development of advanced land navigation system for T-72 battle tanks. He is also the Editor of CRL Technical Journal. His areas of research include signal processing for radar, sonar, navigation systems, radar tracking and image processing applications. 